Tropical Journal of Pharmaceutical Research March 2019; 18 (3): 465-470

ISSN: 1596-5996 (print); 1596-9827 (electronic)

(C) Pharmacotherapy Group, Faculty of Pharmacy, University of Benin, Benin City, 300001 Nigeria.

\title{
Alantolactone exerts anti-proliferative and apoptotic effects on BGC823 and SGC7901 cells via activation of p38MAPK and inhibition of NF-KB signaling pathway
}

\author{
Xie Ningsheng, Xie Junfeng*, Tang Jianhua, Liu Youshun, Wei Yingfeng, Gu \\ Qiuping, Luo Qingtian, Zhu Fangqin \\ Department of Gastroenterology, Ganzhou People's Hospital, Ganzhou City, Jiangxi Province 341000, China
}

*For correspondence: Email: xn26222@163.com

Sent for review: 8 December 2018

Revised accepted: 27 February 2019

\begin{abstract}
Purpose: To investigate the anti-proliferative and apoptotic influences of alantolactone on gastric carcinoma (GC) cell lines, and the mechanism(s) involved.

Methods: Human gastric cancer cell line (BGC823) and gastric adenocarcinoma lymph node metastasis cell line (SGC7901) were maintained in Ham's F12 medium supplemented with $10 \%$ heatinactivated fetal bovine serum (FBS). In each group of cancer cell line, 5 groups of cells were used: control and four alantolactone groups which were treated with increasing concentrations of alantolactone (5 - $30 \mu \mathrm{M})$ for varying periods. Proliferation was determined using MTT assay, while realtime quantitative polymerase chain reaction ( $(\mathrm{R} T-P C R)$ was used to assay the expressions of apoptosis- and metastasis-related genes. The expressions of p38MAPK and nuclear transcription factor-KB (NF-KB) in BGC823 and SGC7901 cells were measured with Western blotting.

Results: Phosphorylated protein (p-p38 protein) expression was significantly higher in both groups of GC cells, relative to control $(p<0.05)$. The expressions of $N F-K B$ in plasma protein were markedly higher in both groups of GC cells than in control group, but the corresponding expressions in nuclear protein were significantly lower in both groups of GC cells, relative to control $(p<0.05)$.

Conclusion: Alantolactone exerts anti-proliferative and apoptotic effects on BGC823 and SGC7901 cells via mechanisms involving activation of the $\mathrm{p38MAPK}$, and inhibition of the NF-KB signaling pathways. Thus, alantolactone may be a new and effective anti-gastric cancer drug.
\end{abstract}

Keywords: Gastric cancer, Alantolactone, Proliferation, Metastasis, Apoptosis

This is an Open Access article that uses a funding model which does not charge readers or their institutions for access and distributed under the terms of the Creative Commons Attribution License (http://creativecommons.org/licenses/by/4.0) and the Budapest Open Access Initiative (http://www.budapestopenaccessinitiative.org/read), which permit unrestricted use, distribution, and reproduction in any medium, provided the original work is properly credited.

Tropical Journal of Pharmaceutical Research is indexed by Science Citation Index (SciSearch), Scopus, International Pharmaceutical Abstract, Chemical Abstracts, Embase, Index Copernicus, EBSCO, African Index Medicus, JournalSeek, Journal Citation Reports/Science Edition, Directory of Open Access Journals (DOAJ), African Journal Online, Bioline International, Open-J-Gate and Pharmacy Abstracts

\section{INTRODUCTION}

Gastric cancer (GC), a malignant tumor of the gastric mucosa is characterized by high incidence and mortality. In China, the incidence of $\mathrm{GC}$ increases on yearly basis due to aging of the population, changing lifestyles, and Helicobacter pylori infection [1]. Clinical studies have shown that lymphatic metastasis of advanced GC is as high as $70 \%$, and proliferation and metastasis of the cells are the main factors responsible for postoperative recurrence of GC [2]. 
An understanding of the metastasis and apoptosis of GC cells is important in achieving improved prognosis. Alantolactone, a sesquiterpene lactone isolated from Inula helenium L., possesses anti-inflammatory, antibacterial and anti-tumor properties, and its anti-tumor effect is particularly prominent [3]. Alantolactone is effective against cancers such as breast cancer, glioma, and multiple myeloma, but studies on its anti-GC activity and possible mechanism of action are scanty [4-6].

The present study was aimed at investigating the anti-proliferative and apoptotic influences of alantolactone on GC cell lines, and the underlying mechanism(s).

\section{EXPERIMENTAL}

\section{Materials and chemicals}

The BGC823 and SGC7901 cell lines were obtained from China Medical University; 3-[4, 5dimethylthiazol-2-yl]-2, 5 diphenyl tetrazolium bromide (MTT) assay kit was purchased from Guangzhou Baiyunshan Pharmaceutical Co., Ltd., while alantolactone was product of Nanjing Jingzhu Biotechnology Co. Ltd. Dimethylsulfoxide (DMSO) was obtained from Tianjin Saifurui Technology Co. Ltd.; ethanol was purchased from Shanghai Youyu Biotechnology Co., Ltd., while Bax, Bcl-2, p53, matrix metallopeptidases 2, 7 and 9 primers were products of Shanghai Jingjing Reagent Co., Ltd. Acrylamide was purchased from Shanghai Yanjin Biotechnology Co. Ltd; ammonium persulfate and bicinchoninic acid (BCA) protein kit were products of Beijing Zhongke Ruitai Biotechnology Co. Ltd, while ECL chemiluminescence liquid was obtained from Beijing Meikewande Biotechnology Co., Ltd. Phosphate buffered saline (PBS) was purchased from SGM Biotech and RIPA protein lysis kit was obtained from Shanghai Spark Crystal Molecular Biotechnology Co. Ltd.

\section{Cell lines and culture}

The BGC823 and SGC7901 cells were maintained in Ham's F12 medium supplemented with $10 \%$ heat-inactivated FBS in a humidified incubator at $37{ }^{\circ} \mathrm{C}$ and $5 \% \mathrm{CO}_{2}$ atmosphere. Cells in exponential growth phase were used for this study. In each group of cancer cell line, the cells were divided randomly into five groups: control group and four alantolactone groups which were treated with increasing concentration of alantolactone $(5-30 \mu \mathrm{M})$ for varying periods.

\section{MTT assay}

Cell proliferation was determined using MTT assay. The cells $\left(1 \times 10^{5}\right.$ cells $\left./ \mathrm{mL}\right)$ were seeded into 96-well plates and pretreated with varied concentrations of alantolactone $(5-30 \mu \mathrm{M})$. This was followed by the addition of $20 \mathrm{~mL}$ of $0.5 \%$ MTT solution within $4 \mathrm{~h}$, after which the culture medium was changed. Then, DMSO (150 $\mu \mathrm{L})$ was added in drops to each well, and the wells were placed for 10 on an oscillator for 10 min so as to ensure dissolution of the formazan crystals. The control wells contained culture medium, MTT solution and DMSO only, and were treated same way as the sample wells. Each well was incubated in the dark for $2 \mathrm{~h}$ and absorbance was measured at $570 \mathrm{~nm}$ using Sunrise enzyme labeling instrument. The procedure was performed in triplicates and cell proliferation was calculated as shown in equation 1.

$\mathrm{C}(\%)=\{(1-A s) / A c\} 100$

where $A s=$ absorbance of sample well, and $A c=$ absorbance of control well.

\section{qRT-PCR}

The treated and control cells were lysed using radioimmunoprecipitation assay (RIPA) buffer. Their total RNAs were extracted using Trizol reagent, and reverse-transcribed to cDNAs, which were then quantified using qRT-PCR. The reaction conditions were: pre-denaturation at 95 ${ }^{\circ} \mathrm{C}$ for $30 \mathrm{~s}$, denaturation at $95{ }^{\circ} \mathrm{C}$ for $5 \mathrm{~s}$, annealing at $60{ }^{\circ} \mathrm{C}$ for $34 \mathrm{~s}$, and 40 cycles. $\beta$ Actin gene was used as internal reference.

\section{Western blotting}

The expressions of p38MAPK), nuclear transcription factor-kB (NF-kB) in BGC823 and SGC7901 cells treated with alantolactone (20 $\mu \mathrm{M}, 24 \mathrm{~h})$ were measured using Western blotting. The supernatant was assayed for protein content with BCA assay. A portion of total tissue protein $(20 \mu \mathrm{g})$ from each sample was subjected to SDS-PAGE, followed by transfer to fixed polyvinylidene fluoride membrane at $110 \mathrm{~V}$ and $90{ }^{\circ} \mathrm{C}$ for $120 \mathrm{~min}$. Subsequently, $3 \%$ BSA in Tris-buffered saline containing $0.2 \%$ Tween20 (TBS-T) was added with gentle shaking at room temperature and incubated to block nonspecific binding of the blot. Thereafter, the blot was incubated with the appropriate primary antibodies (1: 500) at $4{ }^{\circ} \mathrm{C}$ overnight. Then, the membrane was rinsed thrice with TBS-T and further incubated at room temperature with horseradish peroxidase-conjugated secondary antibody for $2 \mathrm{~h}$. The blot was developed using 
an x-ray film. ImageJ software was used for grayscale assessment of the resultant bands. The respective protein expression levels were normalized to that of $\beta$-actin which was used as a standard reference.

\section{Statistical analysis}

Data are expressed as mean \pm SEM, and were statistically analyzed with SPSS (22.0). Group comparisons were carried out with Student $t$-test. Statistical significance was fixed at $p<0.05$.

\section{RESULTS}

\section{Effect of alantolactone on proliferation of GC cells}

The proliferations of BGC823 and SGC7901 cells were significantly inhibited in a time- and concentration-dependent manner by alantolactone, relative to control group $(p<0.05)$. These results are shown in Figure 1.

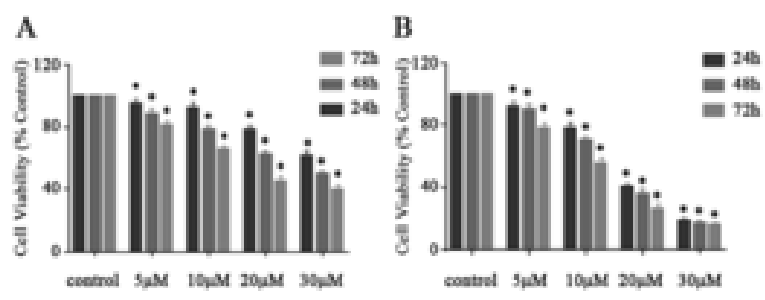

Figure 1: Effect of alantolactone on the proliferation of BGC 823 and SGC 7901 cells. A: BGC823 cells; B: SGC7901 cells. $p<0.05$, relative to control

\section{Levels of expressions of apoptosis-related genes in GC cells}

Figure 2 shows that when compared with control, Bax was expressed markedly higher in BGC823 cells, and its expression increased with time of incubation $(p<0.05)$. In contrast, Bax expression levels in the alantolactone groups and control group were comparable $(p>0.05)$. The expression of $\mathrm{Bcl}-2$ was significantly lower in both groups of GC cells than in control group, and was significantly reduced with time of incubation $(p<0.05)$. The expression of p53 was markedly upregulated in both groups of GC cells, relative to the control group, and was significantly increased with duration of incubation $(p<0.05)$.

\section{Influence of alantolactone on metastasis of GC cells}

As shown in Figure 3, the expressions of the matrix metallopeptidases were significantly lower in both groups of $\mathrm{GC}$ cells than in control group, and were significantly reduced with time $(p<$ 0.05).

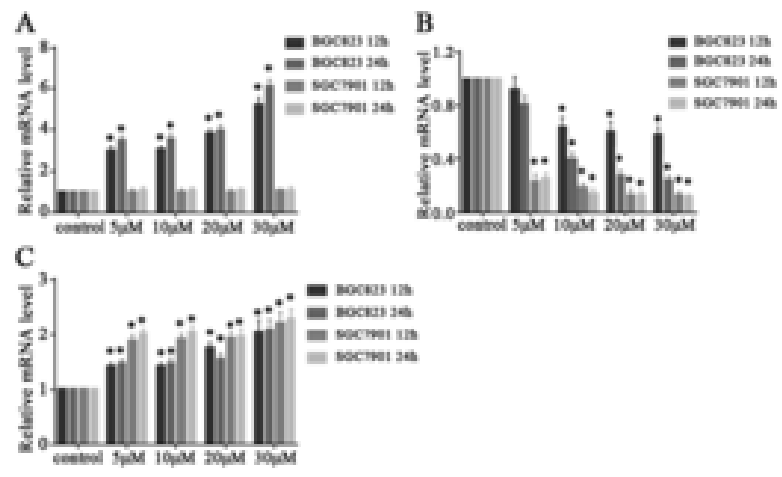

Figure 2: Effect of alantolactone on the expressions of apoptosis-related genes in GC cells. A: Bax; B: Bcl-2; C: p53. $p<0.05$, relative to control

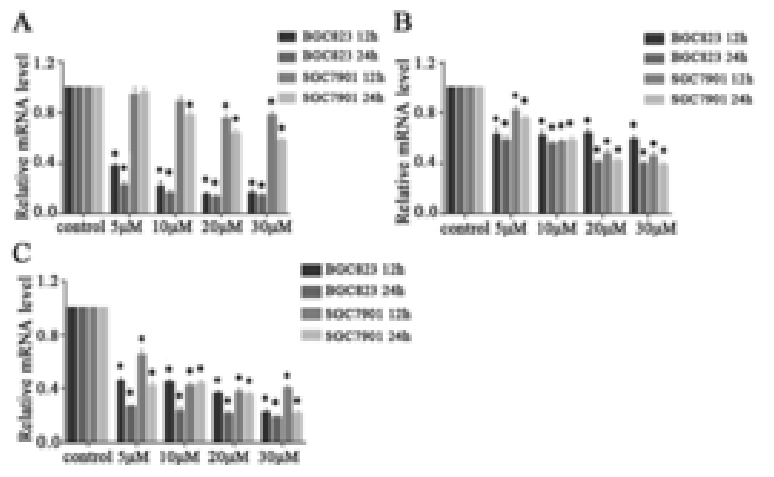

Figure 3: Influence of alantolactone on metastasis of GC cells. A: MMP-2; B: MMP-7; C: MMP-9; ${ }^{\star} p<0.05$, relative to control

\section{Expressions of p38MAPK and p-p38 in GC cells}

The expression levels of p38 protein in the alantolactone groups and control group were comparable $(p>0.05)$. However, phosphorylated protein (p-p38 protein) expression was significantly upregulated in both groups of $\mathrm{GC}$ cells, when compared to control $(p<0.05)$. These results are depicted in Figure 4.

\section{NF-kB expression in GC cells}

The NF-kB protein expression was significantly higher in both groups of GC cells than in the control group, but, compared with control, the corresponding expression of NF-KB in nuclear protein was significantly lower in both groups of GC cells $(p<0.05)$ (Figure 5$)$. However, NF-kB protein expressions in both groups of $\mathrm{GC}$ cells were comparable with that of control group $(p>$ 0.05). 

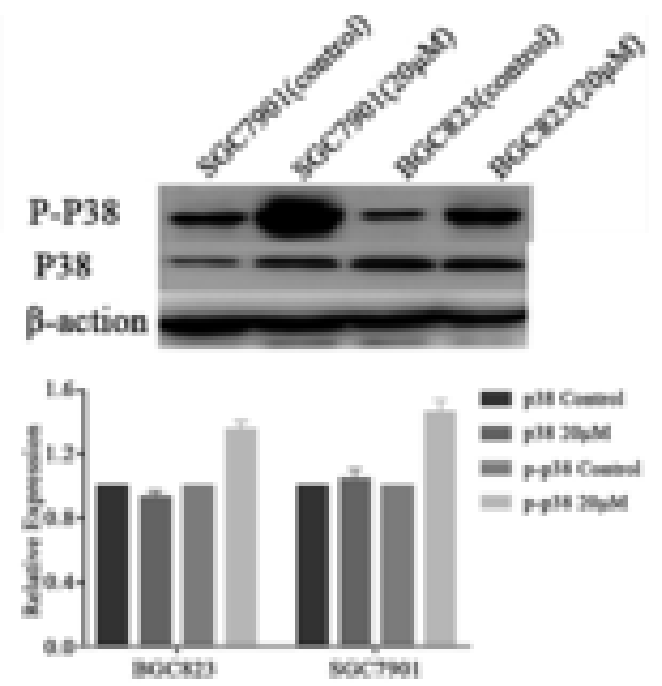

Figure 4: Effect of alantolactone on expressions of p38 protein and p-p38 in GC cells
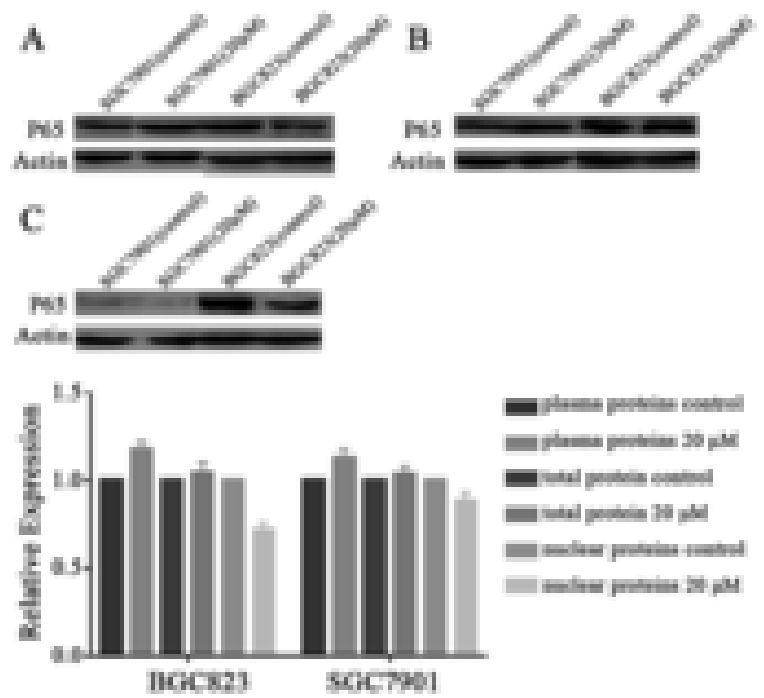

Figure 5: Effect of alantolactone on the expression of NF-KB in GC cells. A: NF-KB expression in plasma proteins of $\mathrm{GC}$ cells; $\mathrm{B}$ : NF-KB expression in total protein of GC cells; C: NF-kB expression in nuclear proteins of GC cells

\section{DISCUSSION}

More than $50 \%$ of GC occur in the antrum of the stomach. It affects the anterior and posterior walls, and small and large curvatures of the stomach. More than $70 \%$ of early GC cases have insidious onset, and as the disease progresses, non-specific symptoms such as upper abdominal fullness, dull pain, pantothenic acid indigestion, nausea and vomiting, and black stool become more prominent [7- 9]. The prognosis of GC is poor due to its complicated pathological mechanism, easy diffusion and metastasis, and single treatment method. Alantolactone is a sesquiterpene lactone isolated from Inula helenium L. It possesses biological activities such as hemodynamic effect, antifungal, anti-tumor, hypoglycemic, and antiinflammatory properties [10]. In vitro studies have shown that alantolactone inhibits the growth of Mycobacterium tuberculosis at a concentration of $0.1 \mu \mathrm{g} / \mathrm{mL}$ [11]. It has been reported that high concentration of alantolactone promotes microvascular perfusion in stimulated rat skeletal muscle [12]. It has also been shown that alantolactone inhibits the proliferation of multiple myeloma RPMI-8226 cells and induces their apoptosis by blocking the activity of ERK signal pathway [13]. In a previous study, it was reported that it inhibits the proliferation and metastasis of mammary cancer MDA-MB-231 cells by blocking the activation of STAT3 signal pathway [14]. Research has shown that alantolactone markedly suppresses proliferation of drug-resistant chronic myeloid leukemia cell lines (K562/ADR and K562), and promotes apoptosis of K562/ADR cells via a mechanism involving mitochondrial apoptosis [15]. Alantolactone produces weak inhibitory effect on the expression of tumor testis antigen TSP50 in normal hepatocytes [16].

In the present study, the proliferations of BGC823 and SGC7901 cells were significantly inhibited, relative to control group, and the inhibition increased with time and increase in the concentration of alantolactone. These results suggest that alantolactone may significantly inhibit the proliferation of GC cells in a concentration- and time-dependent manner, and are in agreement with those previously reported [13-16]. It appears that alantolactone exerts antiGC effect via the inhibition of proliferation and metastasis of the cells.

In this study, Bax expression was markedly upregulated in BGC823 cells, when compared with the control group, and increased with time of incubation. In contrast, Bax expression levels in the alantolactone groups and control group were comparable. The Bcl-2 expression was markedly lower in both GC cell groups than in control group, and the expression was significantly reduced with time of incubation. However, p53 expression was markedly higher in both groups of GC cells than in control group, and was significantly increased with time of incubation. These results suggest that alantolactone may promote apoptosis in GC cells by downregulating $\mathrm{Bcl}-2$ while simultaneously upregulating the expressions of $\mathrm{Bax}$ and p53 genes. The expressions of the matrix metallopeptidases were significantly lower in both groups of GC cells than in control group, and the expressions were significantly reduced with time. This appears to suggest that alantolactone may inhibit the metastasis of GC 
cells by down-regulating the expressions of the three metallopeptidase genes.

It has been reported that p38MAPK is intimately associated with tumor survival and apoptosis, and that it promotes apoptosis in U937 cells by stimulating Bax translocation and enhancing p53 gene expression [17]. In a previous study, it was shown that NF-kB is vital for tumor cell proliferation, metastasis and apoptosis because it controls the expressions of genes for Bax and Bcl-2 [18]. In this study, the results of Western blotting revealed that p38 protein expressions in the alantolactone groups and control group were comparable. However, phosphorylated protein (p-p38 protein) expression was significantly higher in both groups of GC cells than in control group. It is possible that p38MAPK plays an important role in alantolactone-induced apoptosis of $\mathrm{GC}$ cells, and alantolactone may promote the phosphorylation of p38MAPK pathway via activation of downstream apoptosis-related genes p53 and Bax.

In this study, NF-KB expression in plasma protein was significantly higher in both groups of GC cells than in control group, but the corresponding expressions of NF-KB in nuclear protein were significantly lower in both groups of $\mathrm{GC}$ cells than in control group. However, the expressions of $\mathrm{NF}-\mathrm{kB}$ in total protein in both groups of GC cells were not significantly different from that of control group. It is possible that alantolactone prevented the activation of NF-kB by inhibiting its migration to the nucleus of $\mathrm{GC}$ cells, thereby blocking the expressions of the matrix metallopeptidase mRNAs, and inhibiting GC cell metastasis.

\section{CONCLUSION}

Alantolactone exerts anti-proliferative and apoptotic effects on BGC823 and SGC7901 cells via mechanisms involving activation of the p38MAPK pathway and inhibition of the NF-KB signaling pathway. Thus, alantolactone can potentially be developed into a new and effective anti-gastric cancer drug.

\section{DECLARATIONS}

\section{Conflict of Interest}

No conflict of interest associated with this work.

\section{Contribution of Authors}

This work was done by the authors named in this manuscript, and the authors accept all liability resulting from claims which relate to this manuscript and its contents. The study was conceived and designed by Xie JunFeng; Tang JianHua, Liu YouShun, Wei YingFeng, Gu QiuPing, Luo QingTian, Zhu FangQin collected and analysed the data; Xie NingSheng wrote the manuscript. All authors read and approved the manuscript prior to publication.

\section{REFERENCES}

1. Tianwen L, Xiaoyan M, Liyun F, Bingxiu X, Junming $G$. Molecular mechanisms of long noncoding RNAs on gastric cancer. Oncotarget 2016; 7(8): 8601-8612.

2. Heike G, Shivan S, Sally G, Helmut EG, Wolfram M. HER2 expression in gastric cancer: rare, heterogeneous and of no prognostic value - conclusions from 924 cases of two independent series. Cell Oncol 2016; 32 (1-2): 57-65.

3. Wenjun J, Xiaowu T. Advances in anti-tumor research of alantolactone. Mod Med J China 2017; 19(8): 101-104.

4. Lei W, Li T, Fan C, Kun C, Peter P. Terpenes from Carpesium abrotanoides and their cytotoxic activities. Chin Tradit Herb Drugs 2018; 3: 530-535.

5. Wang $X$, Wang $L T$, Zhang B. Effects of alantolactone on migration, invasion and apoptosis of C6 glioma cells. Acta Laboratorium Animalis Scientia Sinica 2018; 3: 1.

6. Yao Y, Yue YS, Dan DX, Ming SN, Kai Z, Zhen YL, Ling $Y Z$, Kai LX. Effect of alantolactone on proliferation of RPMI-8226 cells and its possible mechanism. J Exp Hematol 2015; 23(5): 1336-1340.

7. Qinghua C, Fang L, Kaiyuan J, Ni L, Yuan H, Wenhui Z, Liantang W. MicroRNA-381 inhibits the metastasis of gastric cancer by targeting TMEM16A expression. J Exp Clin Cancer Res 2017; 36(1): 29.

8. Peifei L, Huilin C, Shengcan $C$, Xiaoyan M, Tianwen $L$, Bingxiu X, Rui Y, Junming G. Circular RNA 0000096 affects cell growth and migration in gastric cancer. $\mathrm{Br} \mathrm{J}$ Cancer 2017; 116(5): 626-633.

9. Chaojing L, Zhengxiang S, Chunguang L, Lixin Y. MiR129 regulates cisplatin-resistance in human gastric cancer cells by targeting P-gp. Biomed Pharmacother 2017; 86: 450-456.

10. Junmin Z, Ya L, Dongzhu D, Juan Y, Kun G, Jianguo $F$. Inhibition of thioredoxin reductase by alantolactone prompts oxidative stress-mediated apoptosis of HeLa cells. Biochem Pharmacol 2016; 102: 34-44.

11. Amara M, Tahir M, He Z, Yongming L, Muhammad K, Tonghui M. Alantolactone induces apoptosis, promotes STAT3 glutathionylation and enhances chemosensitivity of A549 lung adenocarcinoma cells to doxorubicin via oxidative stress. Sci Rep 2017; 7(1): 6242.

12. Jae YL, Sang BK, Jaemoo C, Kwang HS, Yeong SK, Suk JC, Hyun JC, In SY, Dae DK. High body clearance and low oral bioavailability of alantolactone, isolated from Inula helenium, in rats: extensive hepatic metabolism and low stability in gastrointestinal fluids. Biopharm Drug Dispos 2016; 37(3): 156-167. 
13. Yonggang F, Zhiyong W, Hongguo G, Jiehua H, Hongyan $W$, Lihua L, Hua L. Isoalantolactone enhances the radiosensitivity of UMSCC-10A cells via specific inhibition of Erk1/2 phosphorylation. Plos One 2015; 10(12): 0145790.

14. Jaemoo C, Rui JL, Mao SC, Yeong SK. Alantolactone selectively suppresses STAT3 activation and exhibits potent anticancer activity in MDA-MB-231 cells. Cancer Lett 2015; 357(1): 393-403.

15. Chunhui $Y$, Hong $C$, Jiangzhou $Y$, Jingbo $Y$, Meiyan $S$, Xiuxiang $M$, Tonghui $M$. Inhibitory effect of alantolactone on the proliferation of K562/ADR cells and its mechanism. Zhonghua Xueyexue Zazhi 2014; 35(6): 515 .
16. $X u$ GM, Zhen BS, Ping $W, Y u W Z$, Lu GS, Yong $L B, Y u$ $Z$, Li HZ, Ying S, Yin W, et al. Alantolactone induces cell apoptosis partially through down-regulation of testesspecific protease 50 expression. Toxicol Lett 2014; 224(3): 349-355.

17. Jing W, Li C, Liang F, Zhenhai Z, Jie S, Dan L, Xiaobin J. Isoalantolactone inhibits the migration and invasion of human breast cancer MDA-MB-231 cells via suppression of the p38 MAPK/NF-KB signaling pathway. Oncol Rep 2016; 36(3): 1269.

18. Jianli L, Meijia L, Shuai W, Yin H, Yapeng H, Zhijun $Y$, Xiangyu C. Alantolactone induces apoptosis and suppresses migration in MCF 7 human breast cancer cells via the p38 MAPK, NF $K B$ and Nrf2 signaling pathways. Int J Mol Med 2018; 42(4): 1847-1856. 